\title{
Adaptive Petri Nets Implementation. The Execution Time Controller
}

\author{
Ramón Piedrafita Moreno, Member, IEEE. José Luis Villarroel Salcedo
}

\begin{abstract}
The reaction time of a controller is a fundamental matter in discrete event control systems. Petri Nets are extensively used in this field. The controller reads the inputs, executes the control Petri net and writes the output in a cyclic manner. The reaction time of this controller depends on the Petri net structure, on the events sequence and on the algorithm that executes the net. With the objective of minimizing the reaction time, we decided to design a Supervisor controller, which we have called Execution Time Controller (ETC). The aim of the ETC is to determine in real time which algorithm executes the Petri Net fastest and to change the execution algorithm when necessary. In the case of systems control, this minimizes the controller reaction time and also minimizes the power consumed by the controller. One possible application of the technique is the minimization of execution time of the Programmable Logic Controllers programs developed in Sequential Function Chart (SFC).
\end{abstract}

\section{INTRODUCTION}

Petri Nets (PN) is a formalism well suited to model concurrent discrete event systems. It has been satisfactorily applied in fields such as communication networks, computer systems, discrete part manufacturing systems, etc. Net models are often regarded as self-documented specifications, because their graphical nature facilitates communication among designers and users. Petri Nets have a strong mathematical basis which allows the validation and the verification of a wide range of correctness and liveness properties. Moreover, these models are executable and can be used to animate and simulate the behavior of the system and also for monitoring purposes once the system is readily working. The final system can be derived from a Petri Net model by means of hardware and software (code generation) implementation techniques. In other words, they can be used throughout the life cycle of a system. In this paper we assume that the reader is familiar with the basic concepts of Petri Nets [1].

In the last 25 years, researchers have devoted considerable attention to the software implementation of PN; see for example [2] [3] [4] [5] [6]. The implementation process is the translation of a system model expressed by a

This work was funded by the NERO project DPI2006-07928 of the Spanish Ministry of Science and Technology.

R. P. M. Corresponding author is with Aragón Institute for Engineering Research (I3A), University of Zaragoza. 50018, Zaragoza, Spain (34 976 762335; fax: 34976 761914; e-mail: piedrafi@unizar.es).

J. L. V. S. Author is with Aragón Institute for Engineering Research (I3A), University of Zaragoza. 50018, Zaragoza, Spain (e-mail: jlvilla@unizar.es).
Petri net to an actual system that has the same behavior as the model. A software implementation is a program that triggers the firing of the net transitions, observing the marking evolution rules, i.e., it plays the token game. Depending on the criteria, a Petri net implementation can be classified as compiled or interpreted, sequential or concurrent, and centralized or decentralized.

In a centralized implementation, the token player is executed by a single task, which is commonly called the coordinator, and the associated actions can be distributed among a set of tasks to guarantee the concurrence expressed by the net (see for example [3] [7] and [8]). Thus, the coordinator acts as the operating system kernel of a multitasking application. In this kind of implementation the algorithm to determine which transitions are enabled and can fire determines its performance. Several algorithms have been proposed in the literature as brute force, place driven or transition driven.

An analysis of centralized implementation algorithms was carried out in [9]. Brute Force (BF), Enabled Transitions (ET), Static Representing Places (SRP) and Dynamic Representing Places (DRP) algorithms were analyzed. The main ideas obtained in [9] are:

- The implementation of the Enabled Transitions, Static and Dynamic Representing Places algorithms can lead to enormous savings in execution time compared to the Brute Force algorithm.

- If the Static Representing Places algorithm chooses suitable Representing Places, performance is similar to or better than Dynamic Representing Places.

- The choice of the most suitable type of algorithm to execute a Petri Net depends on the Petri Net behavior (effective concurrency vs. effective conflicts).

In conclusion, the best algorithm to implement a Petri net depends on its structure and on its dynamic behavior (making and events). Enabled Transitions is better in nets with few conflicts or with conflicts of small size. Representing Places is better in nets with a high number of conflicts or with conflicts of medium-great size.

In this work we have developed a technique which allows the choice in real time of the most suitable algorithm to execute a Petri Net in accordance with the behavior observed at any time. With this aim in mind, we decided to design a supervisor controller, which we have called Execution Time Controller (ETC). The aim of the ETC is to determine in real time which algorithm executes the Petri Net fastest and to change the execution algorithm when 
necessary.

In the case of system control, this minimizes the controller reaction time and also the power consumed by the controller. One application of the technique is the minimization of execution time of the Programmable Logic Controllers programs developed in SFC language.

The organization of this paper is as follows; in Section II, we review the centralized implementation techniques for PN; in Section III the ETC is introduced; Section IV describes the techniques developed for algorithm execution time estimation; in Section $\mathrm{V}$ the technique evaluation is done. This section describes the tests run to evaluate the estimation techniques and the working of the ETC in real time. Finally, in section VI, we present the main conclusions and suggest future lines of research.

\section{CENTRALIZED PETRI NETS IMPLEMENTATION}

Centralized implementation techniques [7] [10] are codified in a task called the Coordinator. To characterize a centralized implementation, the algorithm to determine which transitions are enabled and can fire is important. Various solutions are available for reducing the costs of the enabling test and subsequently the overload introduced by the coordinator. Depending on the solution chosen, centralized implementation techniques can be classified into any of the following classes [11]:

Brute force approaches. All of the net transitions are tested for firing. Brute Force and Immediate Transit Evolution Model (IT)[12] algorithms do not try to improve the search of the enabled transitions. Under the IT algorithm the transition conditions of all of the net transitions are evaluated one by one. In the case of a fireable transition, it is fired immediately. Works as [13][14][15] belong to this implementation class. In these, Petri nets are translated to programming languages for Programmable Logic Controllers as Ladder or Instructions List.

Place-driven approaches. The algorithm Deferred Transit Evolution Model (DT) [12] include only the testing of the transitions descending from the marked places, improving the Brute Force operation. In DT, for all transitions leading from marked places, the output transitions are evaluated first. Then the ones found to be fireable are executed one by one.

For Static Representing Places and Dynamic Representing Places algorithms, only the output transitions of some representative marked places are tested [3]. Each transition is represented by one of its input places, place called Representing Place and the remaining input places are denominated synchronization places. Only transitions whose Representing Place is marked are considered as candidates for firing.

Transition-driven approaches. A characterization of the enabling of transitions, other than marking, is supplied, and only fully enabled transitions are considered. This kind of technique is studied in works such as [11][16].
As was said in the introduction, an analysis of the features of Petri Net centralized implementation algorithms was carried out in [9], where it is illustrated that Enabled Transitions and Static Representing Places are the algorithms that provide better performances. Both reduce the execution time, optimizing the search for enabled transitions.

In the present work we have implemented several algorithms in which different enabled transition search techniques are developed:

- Brute Force

- Deferred transit evolution model

- Immediate transit evolution model

- Static Representing Places

- Dynamic Representing Places

- Enabled Transitions

ETC has been developed taking into account all these algorithms. However, in this paper we present the results using the better ones: ET and SRP [9].

\section{A. Data Structures}

In a centralized interpreted implementation we need a static data structure which encodes the Petri Net structure and a dynamic one which represents the net state, i.e., the marking. All of the algorithms share the same basic data structure (in which the Petri Net is encoded) with different access possibilities adapted to each technique. Likewise, they have two kinds of lists to make the net evolve: treatment lists to be processed in the present treatment cycle and formation lists to be processed in the next cycle. Using these two lists, the net is executed by steps, avoiding the appearance of avalanche effects. The fundamental difference between each of the implementation techniques lies in the way in which the formation lists are built, and hence in the transitions which are considered in each treatment cycle.

In the Enabled Transitions technique the following data structures will be available:

- Enabled Transitions List (ETL). Treatment list containing the transitions with all input places marked.

- Almost Enabled Transitions List (AETL). Formation list formed by the output transitions of the places that will be marked by the firing of the transitions. Hence, AETL contains the transitions which can be enabled in the next cycle.

In the Static Representing Places technique, the following data structures will be available:

- $\quad$ Marked Representing Places list (MRPL) and Marked Synchronization Places list (MSPL). Treatment Lists with the marked Representing Places and Synchronization Places.

- Marked Representing Places list next cycle (MRPLnext) and Marked Synchronization Places list next cycle (MSPLnext). Formation Lists with the Representing Places and Synchronization Places that will be marked in the next cycle by the firing of the transitions. 


\section{B. Algorithm Execution Cycle}

Program 1 presents the basic treatment cycle of the Coordinator for the ET technique and Program 2 for the SRP technique. The code of the actions associated to the net places has been omitted in these programs.

In the ET technique, ETL contains all enabled transitions at the beginning of the cycle. From this list the fired transitions and the disabled transitions (effective conflicts) must be extracted in the execution cycle. AETL is built with the output transitions of the places that will receive tokens after the firing of the transitions. When ETL is updated for the next cycle, the enabling of the transitions in AETL is verified.

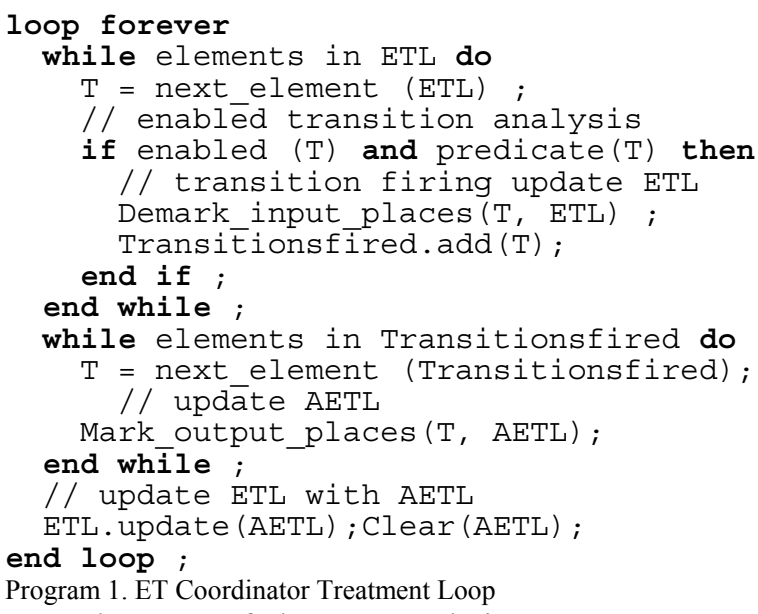

In the case of the SRP technique, MRPL contains the marked representing places and the MSPL the marked synchronization places. The output transitions of a marked representing place are verified for enabling If a represented transition fires the verification process ends because the rest of represented transitions become disabled (effective conflict). MRPLnext and MSPLnext are built with the places that become marked in a treatment cycle. Finally, MRPL and MSPL are incremented with MRPLnext and MSPLnext respectively.

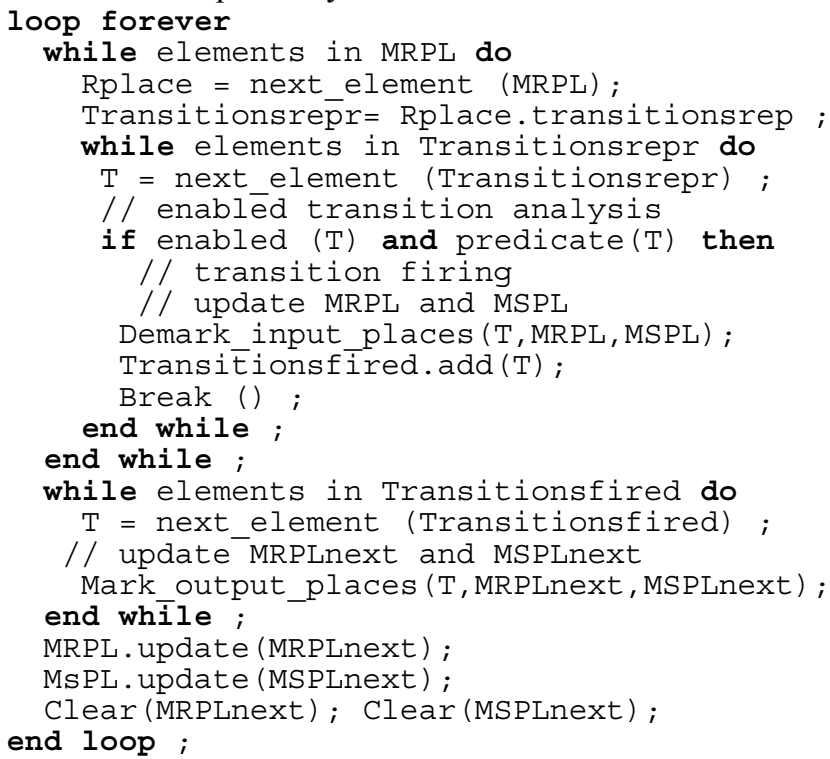

\section{The EXECUTION TIME CONTROLLER}

The choice of the most suitable type of algorithm to execute a Petri Net depends on the Petri Net behavior (effective concurrency vs. effective conflicts). Enabled Transitions is better in nets with few conflicts or with conflicts of small size. Representing Places is better in nets with a high number of conflicts or with conflicts of mediumgreat size [9].

With a view to minimizing Petri Net execution time, we propose an adaptive implementation that will choose the best algorithm to execute the net. We refer to this solution as the Execution Time Controller (ETC) (see Fig. 1). The main function of the ETC will be to determine in real time which algorithm executes a Petri Net fastest. The ETC will execute the algorithm chosen and estimate the execution time of the other non-executed algorithms, choosing the best algorithm in line with the controlled system. If necessary, the ETC changes the algorithm. In the next section we present in detail how the execution time (ExT) of the running and the alternative algorithms are estimated. To avoid the overload of continuous algorithm changes, an integral cost function is used:

$$
\begin{aligned}
& \mathcal{E}=E x T_{\text {calculated }}(\text { running_a } a \lg )-E x T_{\text {estimated }} \text { (alternative_alg) } \\
& I(k)=\left\{\begin{array}{c}
I(k-1)+\varepsilon(k) \text { if } I(k-1)+\varepsilon(k)>0 \\
0 \text { if } \quad I(k-1)+\varepsilon(k) \leq 0
\end{array}\right.
\end{aligned}
$$

The change is made when $\mathrm{I}(\mathrm{k})$ is greater than the half of the execution time of the executed algorithm. When a change happens, I (k-1) $=0$.

// Offline Control

Load Petri Net

Measuring Times

First Choice of the best algorithm

Return to initial mark

// Online Control

loop forever

Read Inputs

Petri Net execution with the best algorithm

Write Outputs

Compute execution time of running_alg

Estimate execution time of alternāte alg

Compute $I(k)$

If $I(k)>\left(\operatorname{ExT}_{\text {calculated }}(\right.$ running_alg $\left.) / 2\right)$ then

Change algorithm

Initialize data structures

$I(k-1)=0$

End if

Wait for next period();

end loop

Program 3. Execution Time Controller

\section{ESTIMATION OF ALGORITHM EXECUTION TIME}

Centralized implementation techniques are based on a cyclic treatment (see Program 1 and 2). The main loop goes through the treatment and formation lists using an algorithm that depends on the executed technique. The coordinator cycle execution time depends on the size of the treatment and formation lists. The size of the treatment lists in the case of ET and SRP depends on the current net marking. It determines the number of enabled transitions and the 
number of marked representing places. The size of the formation lists depends on the number of transitions that fire in the cycle. Thus, the execution time depends on the evolution of the net marking and on the active net part, the net structure and the sequence of events. As algorithms use different lists, their execution times will be different. The estimation of the algorithm execution time is based on the measurement of the mean time taken by these loops and on the estimation in real time of the size of the treatment and formation lists.

First, we study the SRP algorithm. The cycle execution time (CET) can be estimated by the following expression:

$$
\begin{gathered}
\text { CET(SRP) }=\text { T } 1 * \operatorname{SIZE}(\text { MRPL })+\mathrm{T} 2 * \text { FTNUMBER + } \\
\text { T3*SIZE(MRPLNEXT) }+ \text { T4*SIZE(MSPLNEXT) }
\end{gathered}
$$

Where FTnumber is the number of fired transitions; T1 is the mean consulting time of descending transitions from a marked representing place; $\mathrm{T} 2$ is the mean time for firing an enabled transition; T3 is the mean update time of MRPL with a place of MRPLnext and; T4 is the mean update time of MSPL with a place of MSPLnext

The ET algorithm is also analyzed. The cycle execution time can be estimated by the following expression:

$$
\begin{gathered}
\mathrm{CET}(\mathrm{ET})=\mathrm{T} 5 * \operatorname{SIZE}(\mathrm{ETL})+\mathrm{T} 6 * \text { FTNUMBER }+ \\
\mathrm{T} 7 * \operatorname{SIZE}(\mathrm{AETL})
\end{gathered}
$$

Where $\mathrm{T} 5$ is the mean time of the enabling test of a transition; T6 is the mean time for firing a transition and; T7 is the mean update time of ETL with a transition of AETL
Establishing expressions for other implementation techniques is not complicated. Let us consider, for example, the brute force technique. The cycle execution time expression of $\mathrm{BF}$ algorithm is:

$$
\mathrm{CET}(\mathrm{BF})=\mathrm{T} 5 * \operatorname{size}(\mathrm{TL})+\mathrm{T} 6 * \text { FTnumber }
$$

$\mathrm{TL}$ is the list with all transitions of the net. Times T1 to $\mathrm{T} 7$ are measured in an offline execution test. For this purpose, the required measurement instrumentation is incorporated into the program. This instrumentation comprises the instructions required for reading the real time system clock and the necessary time difference calculations.

However, the execution time of the executed algorithm can be measured by reading the system clock. To avoid the overload of the control actions, the execution time of the executed algorithm (runnig_alg) is then calculated with equation (2) to (4) (this depends on the algorithm being executed). In this case FTNUMBER and the sizes of lists (MRPL, MRPLNEXT, ...) are known by the ETC.

The execution times of the alternative algorithms (alternative_alg) must be also estimated with equation (2) to (4). In this case FTNUMBER is also known (the same as in the runnig_alg) but the size of the other lists must be estimated.

For example, in the execution of the ET algorithm the size of the lists of the SRP algorithm must be estimated. The mean number of marked representing places is more or less constant in most nets; therefore, the size (MRPL) will be the mean value estimated in the offline time measurement test.

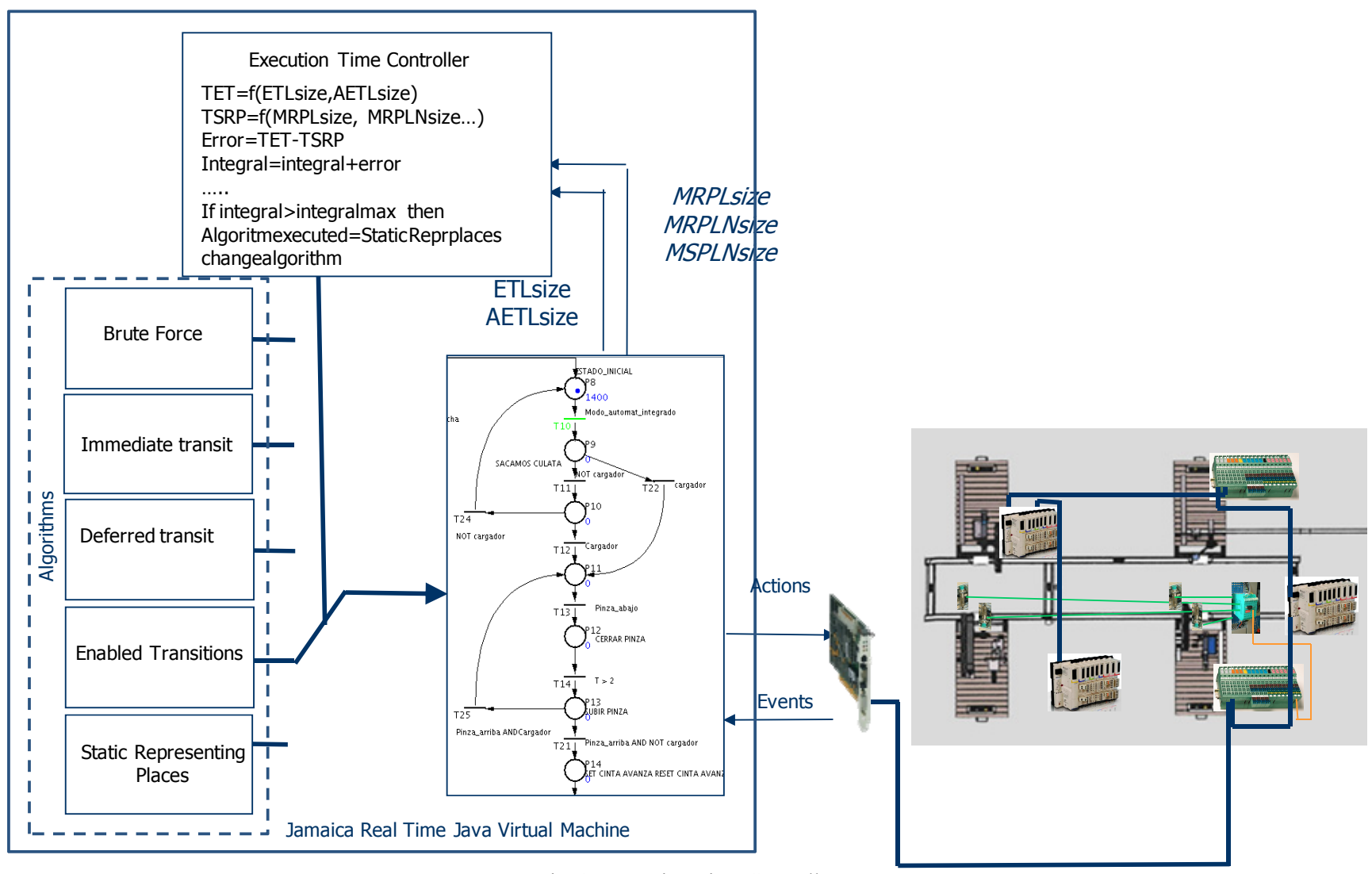

Fig. 1. Execution Time Controller 
Consequently, it can be stated that, on average, the firing of a transition involves the unmarking of its representing place and the marking of a new one. The size (MRPLNEXT) can be approximated by the number of transitions fired.

$$
\text { size }(\text { MRPLNEXT) } \approx \text { FTNUMBER }
$$

The size(MSPLNEXT) can be approximated by the expression:

$$
\operatorname{size}\left(\text { MSPLNEXT) } \approx \text { FTNUMBER } *\left(\mathrm{f}_{\mathrm{p}-1}-1\right)\right.
$$

Where $\mathrm{fp}$ is the mean parallelism factor (number of output places of a transition) of the net.

The goodness of these estimators is analyzed in the following section.

\section{V.TECHNIQUE EVALUATION}

\subsection{Test Platform}

A library of Petri Nets has been developed for carrying out the tests. The library is based on eight base models which can be scaled using a parameter. Some of these models are well-known and frequently used in literature. The library comprises the following nets:
- SEQ. Petri Nets with one sequential process with ne (1..100) states (Fig. 2.a).

- PAR. Petri Nets with p (1..100) sequential processes with 2 places (Fig. 2.b).

- PR1. Petri Nets with p (1..40) sequential processes with 2 states and a common resource (Fig. 2.c). These belong to $\mathrm{s}^{3}$ pr net class [17].

- $D B$. Petri Nets of b (5..11) databases (Fig. 2.d) [18].

- P1R. Petri Nets with 1 sequential process and r (1..40) resources (Fig. 2.e). These belong to $\mathrm{s}^{3} \mathrm{pr}$ net class [17].

- $P H$. Petri Nets with the philosophers' problem [19]. (Fig. 2.f) with $\mathrm{f}(5 . .40)$ philosophers.

- SQUARE. Petri Nets with r (5..15) sequential processes of $\mathrm{r}+1$ states and $\mathrm{r}$ common resources (Fig. 2.g, defined by the authors).

- PR5. Petri Nets of $\mathrm{p}$ (5..62) sequential processes of 6 states and 5 common resources (not shown in figure).

We have implemented the techniques presented in this paper in Java language using Java Real-time extension [20] and following some ideas presented in [8][21]. In our implementations, we used the Real Time Java Virtual Machine JamaicaVM v2.7 [22]. The target hardware was a personal computer with Pentium IV processor at $1.7 \mathrm{GHz}$, running Red Hat Linux 2.4 a)

SEQ

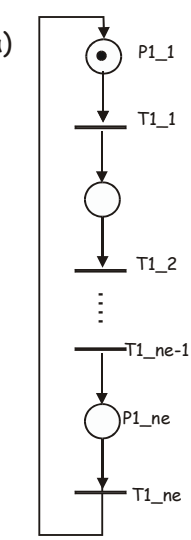

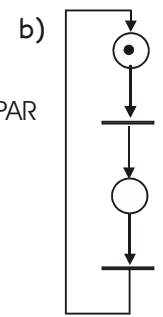
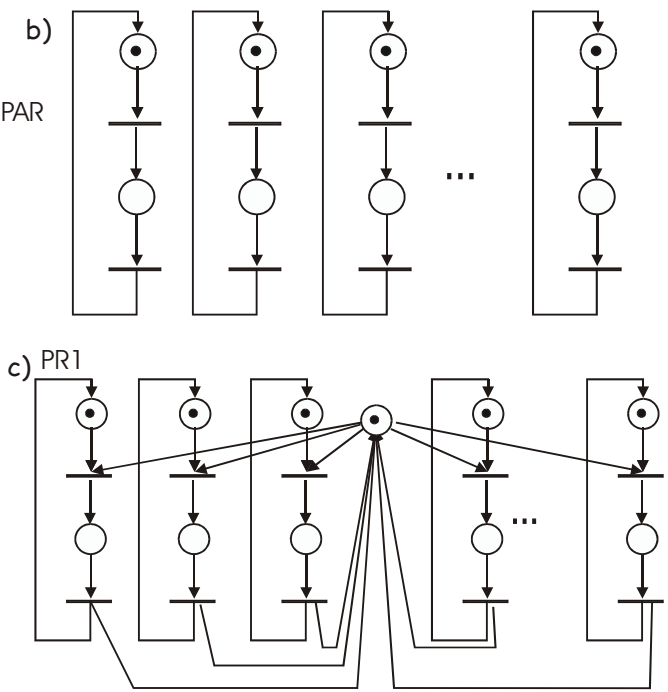

f) $\mathrm{PH}$

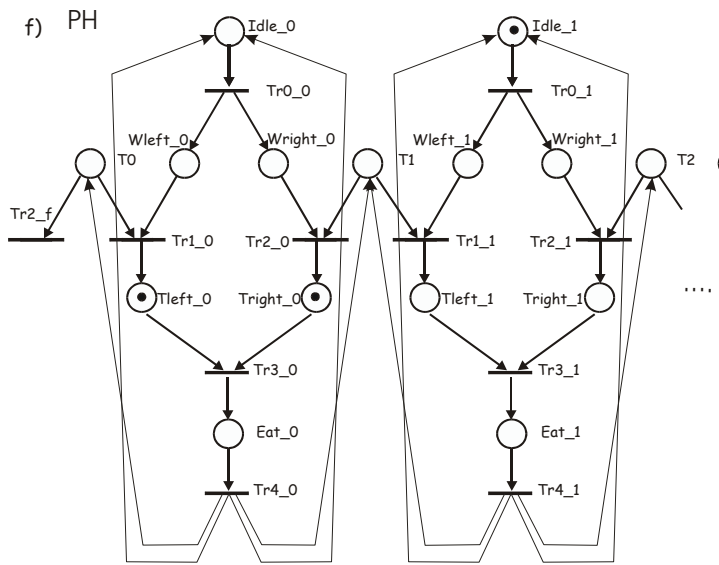

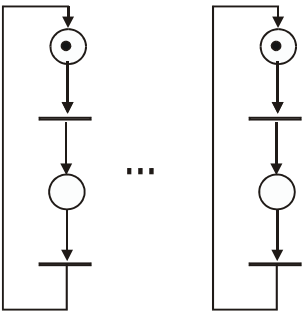

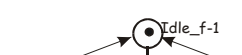

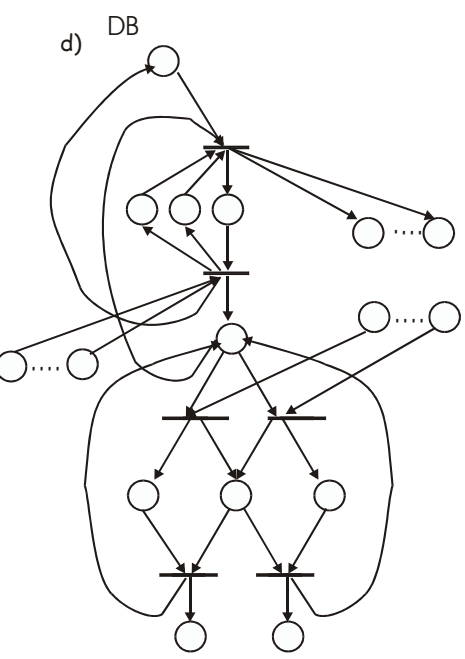

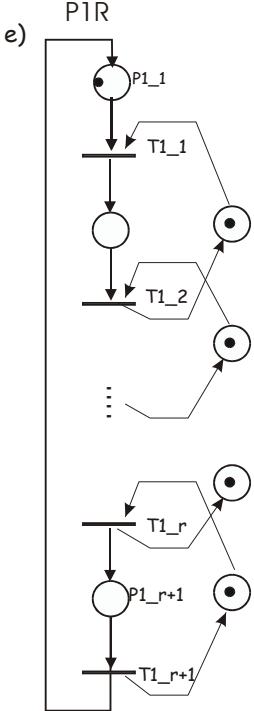

SQUARE

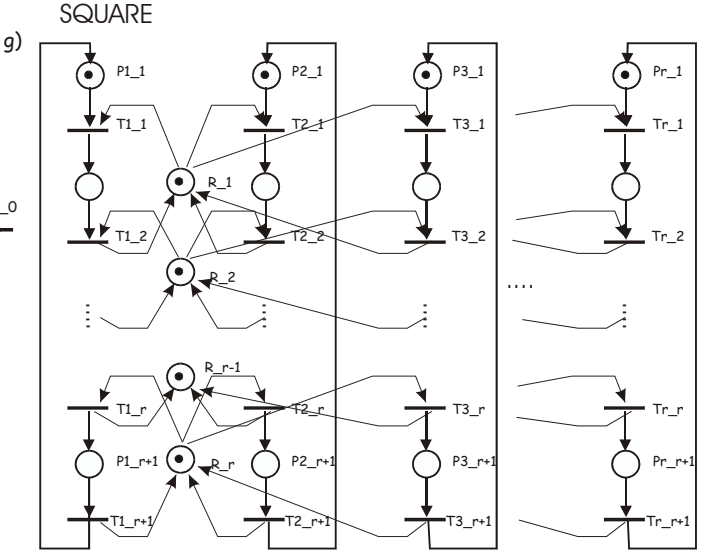

Fig. 2. Petri Nets Library 


\subsection{Execution Time Estimation Tests}

Each test consists of the execution of a Petri net with one of the implemented algorithms. The test concludes when 2000 transitions have fired, obtaining the total execution time of the coordinator. Each net is also executed with the others implemented algorithms.

The results of the tests carried out to establish the precision of estimations are shown in Fig. 3. Figures a..e show the estimation of the execution time compared to the measured execution time for the two algorithms analyzed and for several kinds of nets (SEQ, PAR, PR5, SQUARE, $\mathrm{PH})$, varying the characteristic parameter of each net. As can be seen in the figures, in all tests the estimation has been precise enough. The error is normally smaller than $7 \%$.
On the other hand, Fig. 3.f to Fig. 3.i show four examples of the overload introduced by the estimation of the execution time of the alternative algorithm. In all performed tests the overload is minimal.

\subsection{Real Time Execution of ETC}

The ETC controller has been tested with all the nets in the library and also with a real control application. This is a Flexible Manufacturing Cell in the Computer Science Department of the University of Zaragoza. The ETC obtains a high degree of success in all of the performed experiments, but here for sake of brevity we present the results of the three representative experiments. However an exhaustive report of the experiments can be consulted in [23], accessible via the Internet.

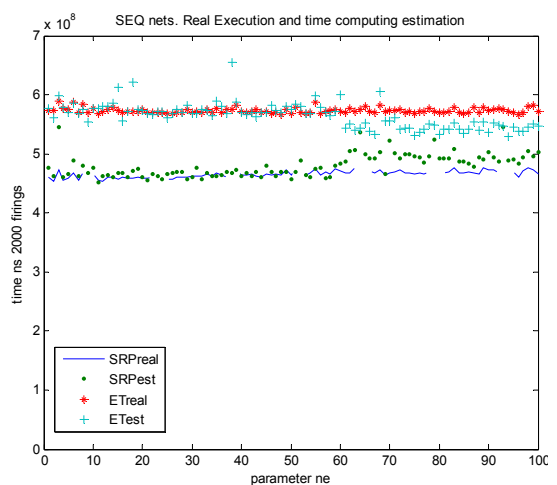

a)

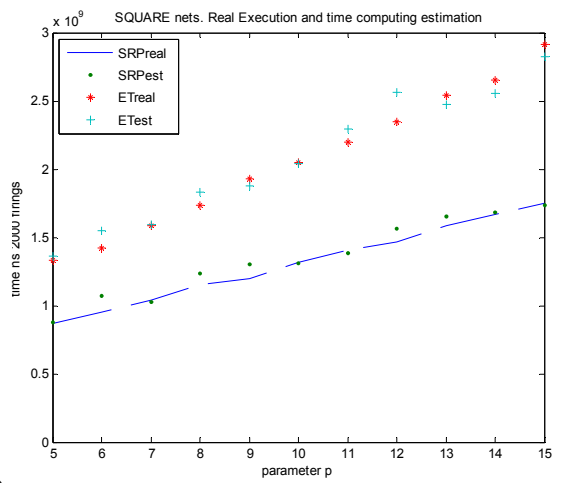

d)

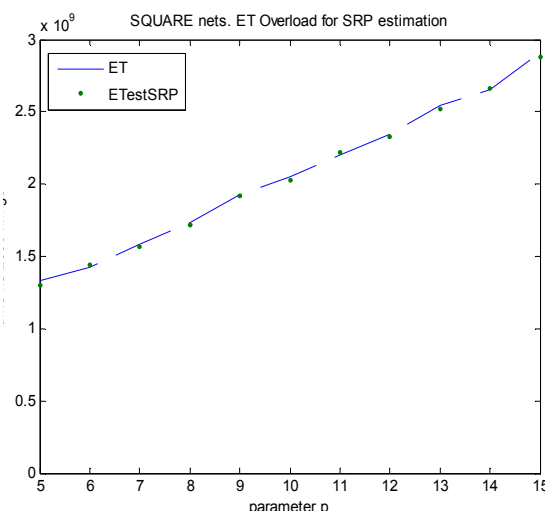

g)

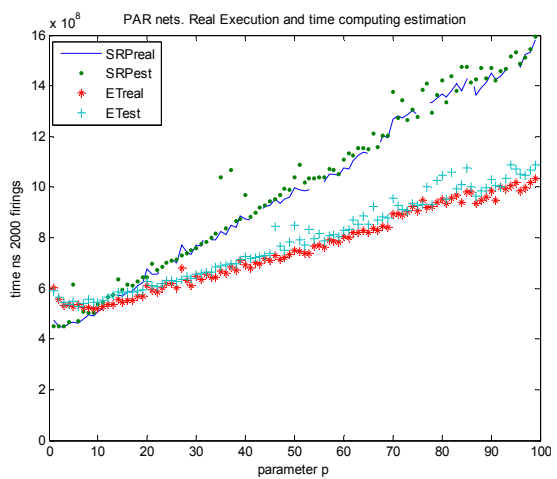

b)

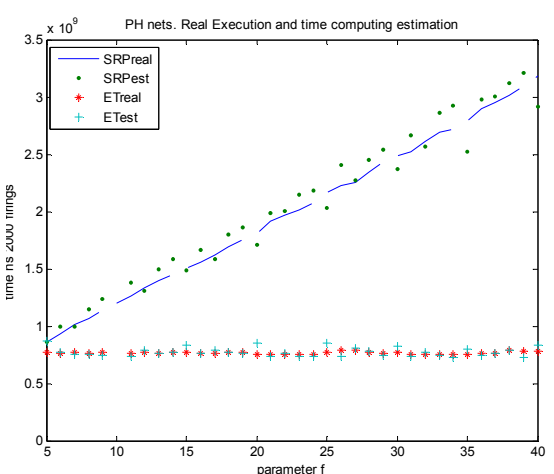

e)

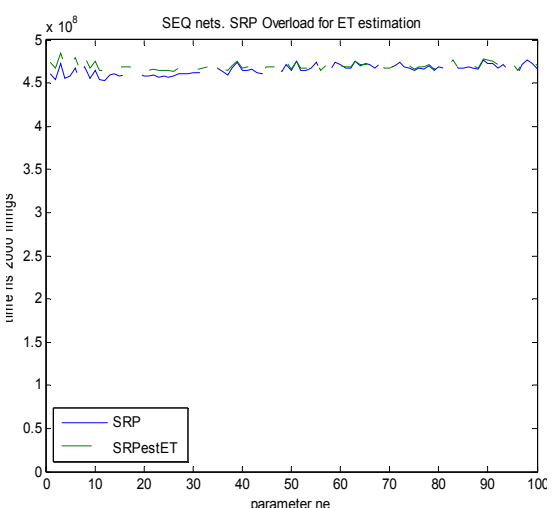

h)
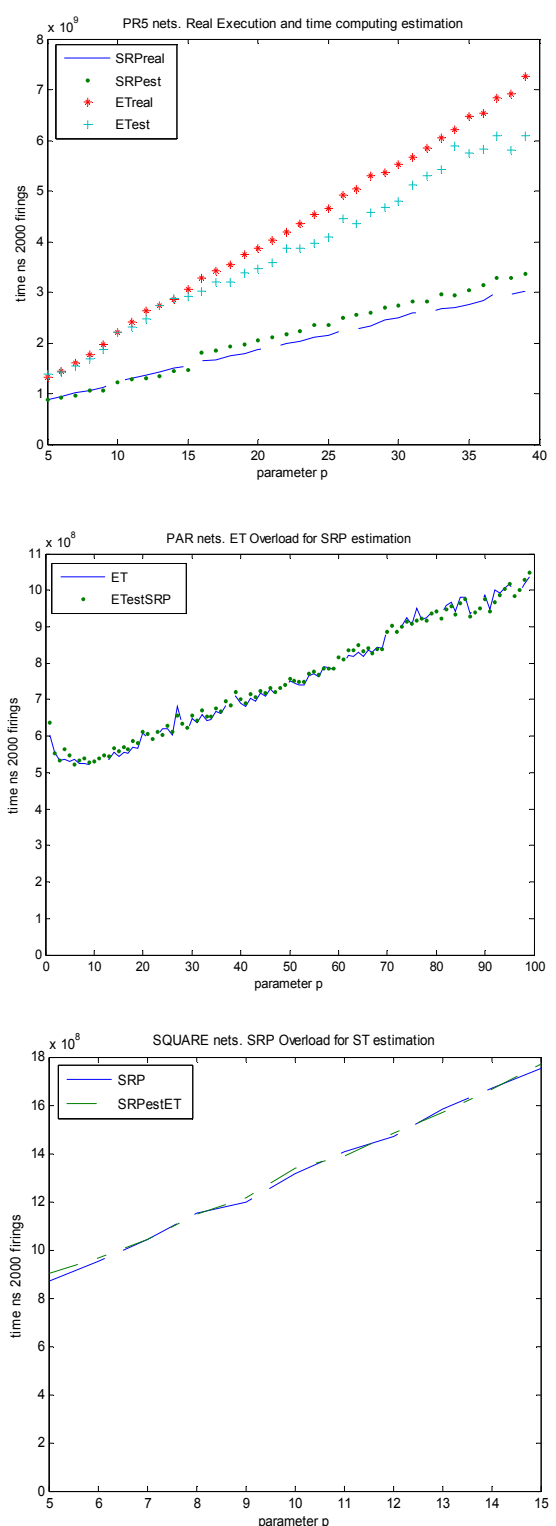

Fig. 3. Petri Net tests with ET and SRP. SRPreal and ETreal are the real execution times of algorithms and SRPest and ETest the estimated ones. In figures $\mathrm{f}$ and $g$ the plot ET is the execution time of the algorithm without performing the estimation of SRP and ETestSRP with estimation. In figures $h$ and $i$ the plot $\mathrm{SRP}$ is the execution time of the algorithm without performing the estimation of ET and SRPestET with estimation. 
The execution of the ETC takes place in the Real Time Java Virtual Machine Jamaica. This is implemented as Periodic Real Time Thread of high Priority with $20 \mathrm{~ms}$ of period. The execution is made in a single processor and threads are scheduled following a static priorities policy without round-robin. The first experiment that we present is over a SQUARE net (see Fig. 2.g) of 15 resources. Fig. 4 shows the results of this experiment and illustrates that the SRP algorithm is always the best [9].

Fig. 4.a, Fig. 5.a and Fig. 6.a show the Real Time execution of The Execution Time Controller (ETC), the Real Time execution of SRP algorithm, the Real Time Execution of ET algorithm, and the integral cost function I(k).

ETC chooses from the beginning the SRP since the estimation of the execution time of this algorithm is smaller than the one of ET algorithm. Because SRP is always better than ET, the integral cost function I(k) (see Fig. 4.a) remains null forever and then no algorithm change is performed. This choice supposes an execution time saving of $37 \%$. For example, in the first 2.5 seconds the ETC saves 600 milliseconds (see Fig. 4.b).
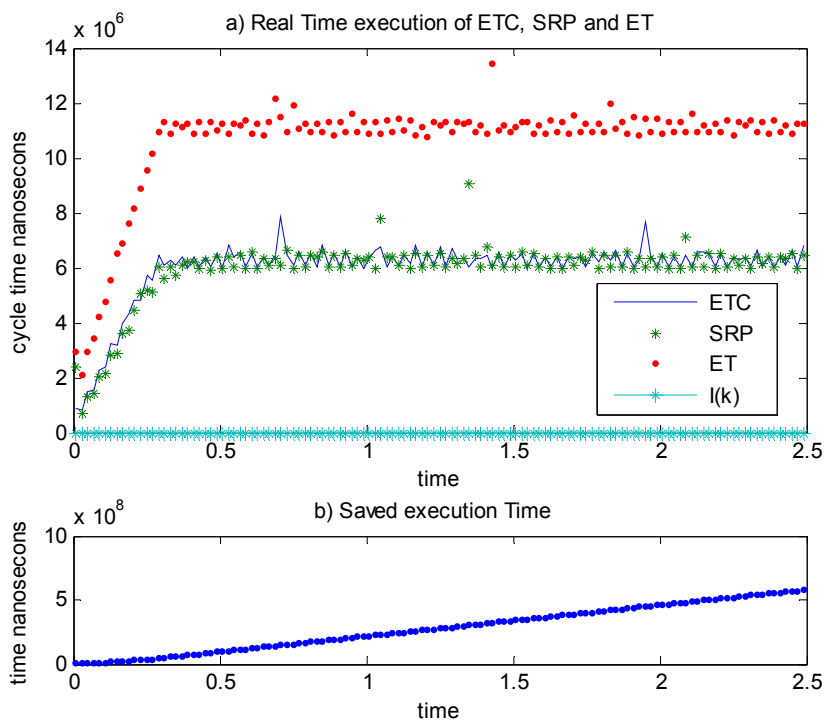

Fig. 4 Execution of Petri Net Square of 15 resources with the ETC

The next experiments were carried out on a hybrid net comprising a SQUARE of 8 resources and a PAR of 20 processes (see Fig. 2.g and Fig. 2.b). In this net, if events reach both the SQUARE and the PAR parts in similar amount, both the SRP and ET algorithms show the same execution time. If more events reach the SQUARE part of the net, the algorithm with better behavior is the SRP (see Fig. 3.d). Otherwise, if more events reach the PAR part, the ET algorithm provides a smaller execution time (see Fig. 3.b). That is, the Petri net has parts with different behaviors.

Fig. 5 shows a test in which events reach only the SQUARE part for 0.4 seconds (the best algorithm will be SRP) and in the following 0.8 seconds, events reach only the PAR part of the net (the best algorithm will be ET). The event sequence is cyclically repeated.
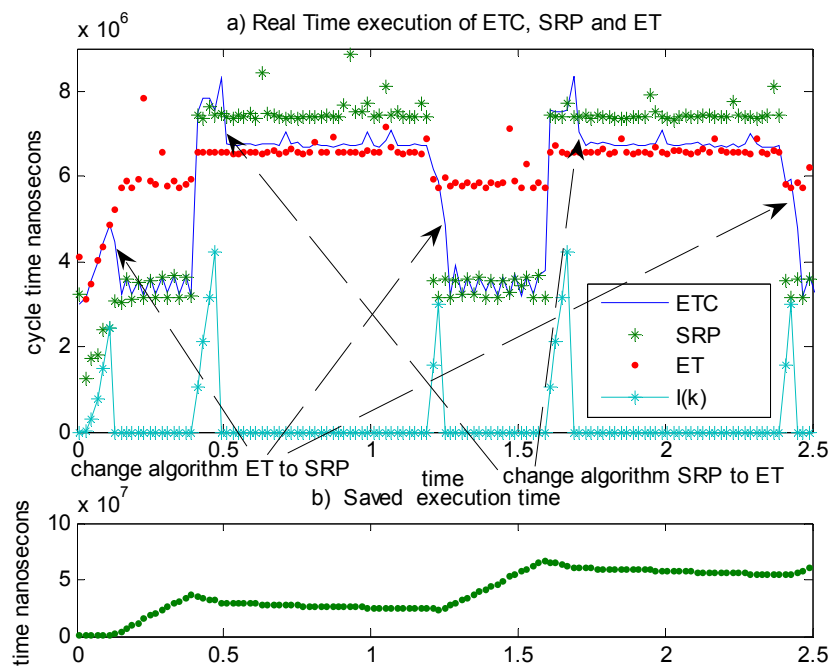

Fig. 5 Execution of Petri Net Square 8 and PAR 20 with the ETC

At the beginning, the ETC executes the ET algorithm. But, as SRP has better execution time, $\mathrm{I}(\mathrm{k})$ increases and in instant 0.14 ETC changes ETC to SRP algorithm. At instant 0.4 ET becomes better, therefore $\mathrm{I}(\mathrm{k})$ increases again and the ETC changes to ET at instant 0.49 . For whole evolution, ETC changes to SRP at instants $0.14,1.26$ and 2.46 and to ET at instants 0.49 and 1.7.

In switching instants, the treatment list of the new algorithm must be calculated. Obviously, it supposes an overhead in computation time of the implementation. Figure 5 shows these switching instants. Only in the change from SRP to ET algorithm, the overhead can be identified as a small pick in the cycle time of ETC. Another possibility would be that the ETC maintains the lists of the all alternative algorithms. This option has been discarded because the overload is greater than the possible time saving.
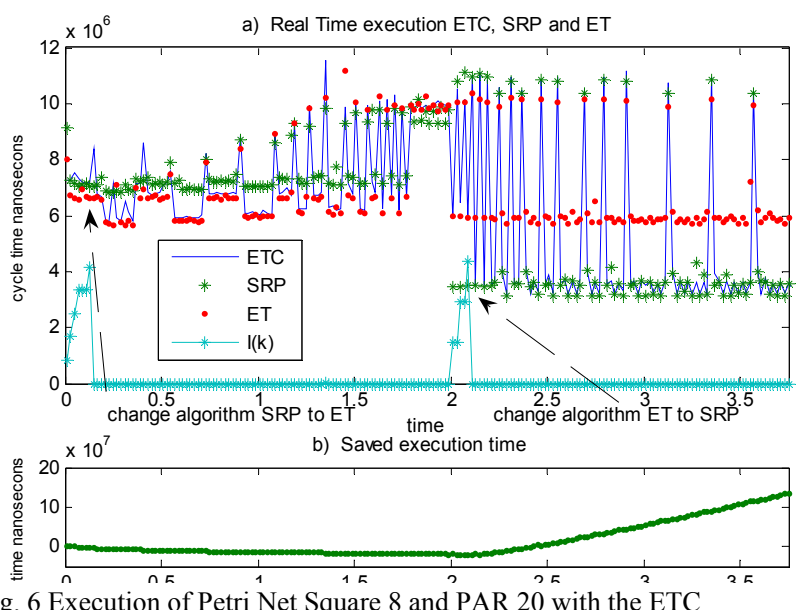

Fig. 6 Execution of Petri Net Square 8 and PAR 20 with the ETC
Fig. 6 shows the last experiment that we present. The same hybrid net as the previous one is used but now, more events reach the PAR part in the first 1.8 seconds (the best algorithm will be ET); the events that reach the SQUARE part are gradually increased and between 1.8 and 2 seconds the two algorithms behave practically identically. Finally, from second 2 more events reach the SQUARE part (the 
best algorithm will be SRP). As can be seen, ETC also behaves correctly. Therefore, ETC chooses in real time the best algorithm to execute the Petri net. The integral function introduces some delay in changes, but it prevents the changing from one algorithm to the other in each cycle. Thus, the $\mathrm{I}(\mathrm{k})$ function acts as a low pass filter.

Fig. 4.b, Fig. 5.b and Fig. 6.b show the saved execution time. When it is positive we have a saved execution time and when it is negative, a slight overload introduced by the ETC with respect to the best algorithm is obtained.

\section{CONCLUSIONS}

In this work we have developed an adaptive Petri net implementation, the Execution Time Controller, which allows choosing in real time the most suitable algorithm to execute a Petri Net. The main function of the ETC will be to determine which algorithm executes a Petri Net fastest. The proposed technique is analyzed with the two most important algorithms (from the point of view of performance): the enabled transitions and the static representing places. However, the ETC can work with any centralized PN implementation algorithm.

The ETC executes the chosen algorithm and estimates the execution time of other non-executed algorithms, deciding the best one in line. The execution of a Petri Net without a suitable algorithm can lead to a significant increasing of the execution time, together with a less satisfactory and slower answer in control applications. The technique has been tested on wide nets with several structures and behaviors. Moreover the ETC has also been tested in a real control application. The technique presents a high success rate in the choice of the best implementation algorithm. The execution of the ETC can lead to enormous savings in execution time; in an example presented, the saving is $37 \%$, but with other nets the saving can be up to $70 \%$.

On the other hand, the ETC allows faster reaction time in Petri Net-based control systems and also minimizes the power consumed by the controller. One possible application of the technique is the minimization of execution time of the Programmable Logic Controllers programs developed in Sequential Function Chart.

Our future work will concentrate on the following two ideas:

- The incorporation of new Petri Net execution algorithms into ETC.

- The improvement of the real time estimation of the execution time algorithms.

\section{ACKNOWLEDGMENTS}

We want to thank Javier Campos, Cristian Mahulea, José Manuel Colom and Manuel Silva for their helpful aid.

\section{REFERENCES}

[1] T. Murata, "Petri Nets: Properties, Analysis and Applications", Proc. of the IEEE, 77(4), 541-580. 1989.
[2] G.W. Brams, editor. "Reseaux de Petri. Theorie et practique". Masson, 1983.

[3] J.M. Colom, M. Silva, and J.L. Villarroel. "On software implementation of petri nets and colored petri nets using high-level concurrent languages". In Proc of 7th European Workshop on Application and Theory of Petri Nets, pages 207-241, Oxford, July 1986.

[4] J.L. Briz and J.M. Colom. 2Implementation of Weighted Place / Transition Nets based on Linear Enabling Functions". Proc of 15th International Conference on Applications and Theory of Petri Nets. pages 99-118. Univ. Zaragoza, 1994.

[5] D. Taubner. "On the implementation of Petri Nets". Advances in Petri Nets 1988, volume 340 of Lecture Notes in Computer Sciences, pages 418-419, Springer-Verlag, Berlin, Germany, 1988.

[6] G. Bruno, G. Marchetto. "Process-translatable Petri Nets for the rapid prototyping of Process Control Systems". IEEE Transactions on software Engineering, Vol. SE-12, NO.2. pages 346-357. February 1986.

[7] R. Valette, M. Courvoisier, J.M. Bigou, and J. Albukerque. "A petri net based programmable logic controller". In Proc. of IFIP Conference on Computer Applications in Production and Engineering, CAP, pages 103-116, 1983.

[8] R. Piedrafita and J.L. Villarroel. "Implementation of Time Petri Nets in Real-time Java". The 4th International Workshop on Java Technologies for Real-time and Embedded Systems. Paris, 2006.

[9] R. Piedrafita and J.L. Villarroel. "Performance Evaluation of Petri Nets Execution Algorithms". IEEE International Conference on Systems, Man, and Cybernetics. Montreal, 2007.

[10] J.L. Villarroel. "Integración Informática del Control de Sistemas Flexibles de Fabricación”. PhD thesis, Dpto. de Ingeniería Eléctrica e Informática, Universidad de Zaragoza, Septiembre 1990.

[11] J.L. Briz. "Técnicas de implementación de Redes de Petri". PhD thesis, Univ. Zaragoza, 1995.

[12] A. Hellgren, M. Fabian, and B. Lennartson. "On the execution of sequential function charts". Control Engineering Practice. Volume 13, Issue 10, Pages 1283-1293, October 2005

[13] S. S. Peng and M. C. Zhou. "Ladder diagram and Petri-net-based discrete-event control design methods". Systems, Man and Cybernetics, Part C: Applications and Reviews, IEEE Transactions on Volume 34, Issue 4, Page(s):523 - 531, Nov. 2004

[14] M. Uzam and A.H. Jones, "Towards a Unified Methodology for Converting Coloured Petri Net Controllers into Ladder Logic Using TPLL: Part I - Methodology", Proceedings of International Workshop on Discrete Event Systems - WODES'96, pp. 178 - 183, Edinburgh, UK, August 19 - 21, 1996,.

[15] S. Klein, G. Frey and M. Minas. "PLC Programming with Signal Interpreted Petri Nets". Proceedings of the ICATPN 2003, Eindhoven, LNCS 2679. Pp 440-449. Srpinger Verlag, June 2003.

[16] M. Silva and S. Velilla. "Programable logic controllers and petri nets: A comparative study". In Proc. of the Third IFAC/IFIP Symposium on Software for Computer Control 1982, Madrid, Spain, October 1982.

[17] J. Ezpeleta and J.M. Colom and J. Martínez: "A Petri net based deadlock prevention policy for flexible manufacturing systems". IEEE Transactions on Robotics and Automation, Vol. 11, N. 2, pp. 173-184, April 1995

[18] K. Jensen. "Coloured petri nets". In W. Brauer, W. Rdsig, and G. Rozenberg, editors, Petri Nets: Central Models and Their Properties, pages 248-299. Springer-Verlag, 1987. LNCS 254.

[19] E. W. Dijkstra. "Hierarchical ordering of sequential processes". Acta Informática, vol.1, pp. 111-127

[20] The Real-Time for Java Expert Group. "The Real-time Specification for Java”. https://rtsj.dev.java.net/. Addison Wesley, 2000.

[21] R. Piedrafita and J.L. Villarroel. "Petri nets and Java. Real-Time Control of a flexible manufacturing cell". . 11th IEEE International Conference on Emerging Technologies and Factory Automation. Prague, September 2006.

[22] Aicas GmbH. JamaicaVM Realtime Java Technology. http://www.aicas.com/jamaica.html. 2007.

[23] R. Piedrafita. "Experimentation with the Execution Time Controller." (Research Report). Departamento de Informática e Ingeniería de Sistemas. Centro Politécnico Superior, 2007. Available online at http://automata.cps.unizar.es/realtime/ 